\title{
Reversed granulation in mid-photosphere of the Sun
}

\author{
Katja Janssen and Gianna Cauzzi \\ INAF Osservatorio Astrofisico di Arcetri, Largo E. Fermi 5, 50125 Firenze, Italy \\ email: kjanssen@arcetri.astro.it
}

\begin{abstract}
Reversed granulation is predicted by numerical simulations and has been observed in the wings of chromospheric lines. Here we present reversed granulation in high spatial, spectral and temporal resolution, extracted from the photospheric Fe I 7090.4 $\AA$ line core intensities, once taking into account the velocity effects. Correlations with the continuum are studied in terms of smoothing, time delay and temporal evolution. The latter appears to be superimposed with an oscillatory signal that suggests the presence of gravity waves in the mid-photosphere.
\end{abstract}

Keywords. Sun: photosphere, Sun: chromosphere, Sun: granulation, Sun: oscillations

\section{Introduction and data characteristics}

The phenomenon of "reversed granulation", the partial reversal of the granular/ intergranular intensity contrast occurring in the mid-photosphere, is studied by means of a 47 minutes time series of good quality $2 \mathrm{D}$ intensity maps acquired in the photospheric $\mathrm{Fe} I$ $7090.4 \AA$ line, extended by some images in the blue Ca II $8542 \AA$ line wing. We analyze a very quiet internetwork region, in which no obvious magnetic structures are visible.

The investigation presented here was performed with IBIS, the Interferometric BIdimensional Spectrometer (Cavallini 2006), a tunable, double Fabry-Perot system at the NSO/ DST. Timeseries of 141 scans were taken on 2nd June 2004 with the high order adaptive optics (AO) with 97 subapertures in quiet Sun, disc center, in 16 wavelength positions in the Fe I $7090.4 \AA$ line and some positions in the Ca II $8542 \AA$ line wing. The images have a scale of $0.17^{\prime \prime}$ per pixel and a size of $512 \times 512$ pixels. After the application of dark current and flatfield corrections, the variation of the transmission wavelength of IBIS throughout the field of view was taken into account, interpolating all spectral profiles to the same wavelength positions creating thus monochromatic images. Images were tracked in time and a p-mode filter with $\mathrm{c}_{\mathrm{s}}=6 \mathrm{~km} / \mathrm{s}$ was applied in terms of a cubic cut in the $\mathrm{k}_{\mathrm{x}}-\mathrm{k}_{\mathrm{y}}-\omega$ space.

\section{Velocity effect vs. reversed granulation}

The Fe I 7090.4 $\AA$ line clearly shows the transition from granular structure in the wings to reversed granulation in the core (Fig. 1). However, to reveal this phenomenon, it is crucial to take the vertical velocity including p-mode effects into account. The inverted structure usually seen in the blue wing of photospheric lines is due to the velocity effect, i.e. blueshifted granular profiles turn dark while redshifted intergranular profiles are still bright. In the red wing of the average profile granules get brighter already, while intergranular profiles still get deeper, thus increasing the granular contrast. A cancellation of all velocities results in a symmetric behaviour of the spectral positions and reveals the reversed granulation pattern in the line core as seen in Fig. 1. 

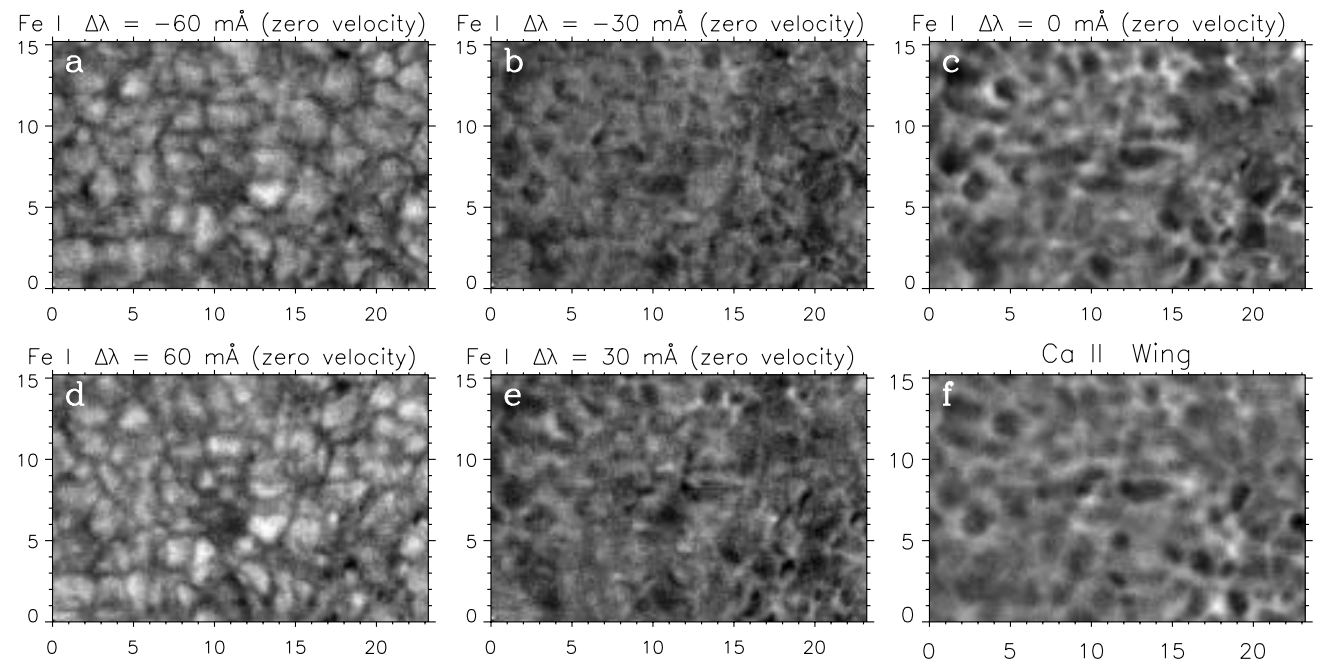

Figure 1. Intensity maps of the Fe I $7090.4 \AA$ line. Spectral positions of $-60 \mathrm{~m} \AA,-30 \mathrm{~m} \AA, 0 \mathrm{~m} \AA$ (upper row), $+60 \mathrm{~m} \AA$ and $+30 \mathrm{~m} \AA$ (lower row) from actual line core, with all spectral profiles shifted to zero velocity, plus CaII $8542 \AA$ at $0.9 \AA$ in the blue wing (lower right). The Fe I line core image (upper right) presents the real 'reversed granulation', comparable to the one seen in the Ca II line wing.

\section{Anticorrelation with underlying continuum}

The quasi-simultaneous observations in a Ca II wing position, basically unaffected by velocity, show the same reversed granulation pattern, but reach higher anti-correlation values with the continuum. The iron line core intensity provides a weak anticorrelation of about -0.21 , while the correlation between calcium wing and continuum reaches -0.32 .

For a spatial smoothing up to $0.5^{\prime \prime}$ the anticorrelation remains constant, but it rapidly decreases if forced to lower resolution, indicating a typical granulation scale for the phenomenon. The anti-correlation increases instead, see Fig. 2, if a time-delay is allowed between the continuum and the higher atmosphere, compare Rutten et al. (2004). This is consistent with the idea of cooling, upflowing granules and consequent radiative heating of gas flowing sideways above intergranular lanes. The strongest anticorrelation is found for a delay of about two minutes for both the Fe I line core and the Ca II wing. The Ca II wing reaches the maximum $10 \mathrm{~s}$ earlier than FeI, confirming that the CaII wings are

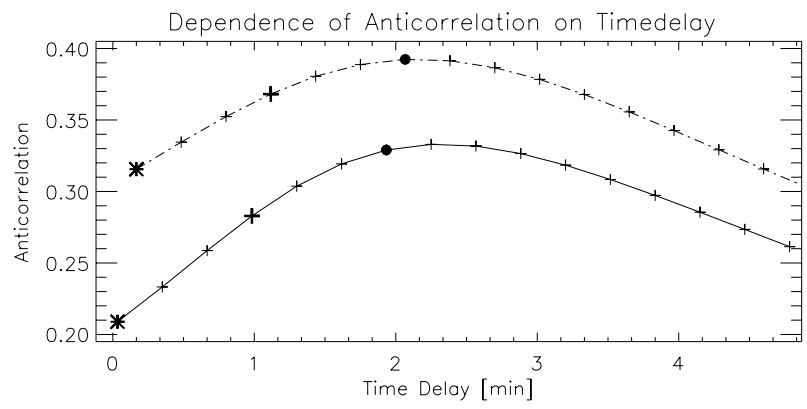

Figure 2. Anticorrelation between continuum and Fe I line core or Ca II wing intensities as a function of their time delay. The anticorrelation, averaged over 141 scans, follows a smooth curve with a maximum of 0.33 for Fe I at 135 s delay (solid line) and 0.39 for Ca II at $126 \mathrm{~s}$ delay (dashed dotted). The thick symbols (stars, crosses and dots) refer to figure 3. 
formed deep in the photosphere at around $150 \mathrm{~km}$ (Uitenbroek 1989) and possibly even below the Fe I line core.

\section{Temporal variation in anticorrelation}

The temporal evolution of the anti-correlation between continuum and Fe I line core intensities shows a clear oscillatory signal, with a period of about 6 minutes, originating in the higher level (Fig. 3). Such a periodic signal is not obvious in the Ca II wing correlation with the continuum.

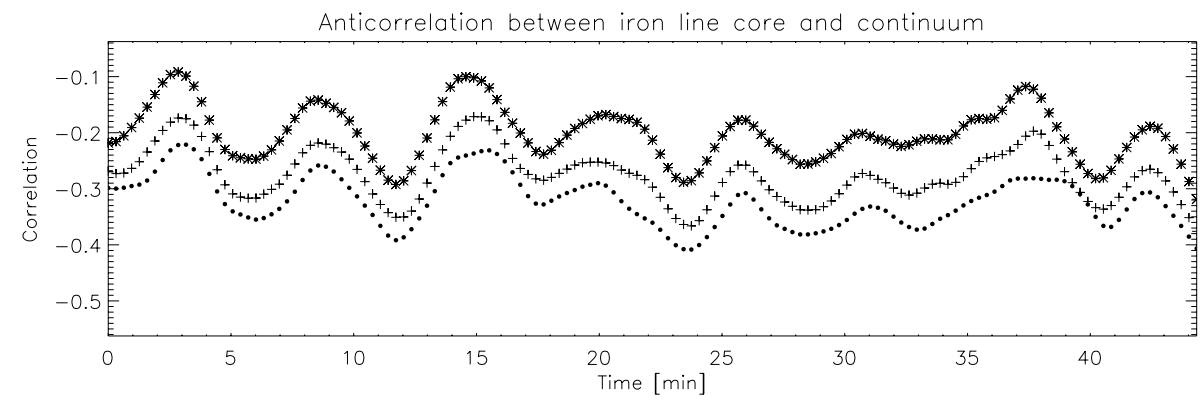

Figure 3. Temporal evolution of the anticorrelation between FeI line core and continuum. The oscillation originates in the higher layers, as a time delay between line core and various continuum images (stars, crosses, dots: 0, 1, 2 minutes) strengthens the anticorrelation, but does not result in a phase shift of the oscillation.

The oscillation persists, even if a stronger p-mode filter with a sound velocity of $5 \mathrm{~km} / \mathrm{s}$ is applied. The period of the oscillation, its presence only in the higher atmospheric layers, and the stronger anticorrelation with the continuum in the lower Ca II wing, are highly suggestive of the presence of gravity waves in the higher, mid-photospheric atmosphere. The reversed granulation pattern, that is strong in the Ca II images and peaks at granular scales, might become "corrupted" by intensity oscillations in mid-photosphere. Indeed, gravity waves are supposed to develop steeply with height (Mihalas \& Toomre 1981), therefore at the height of the FeI core formation their amplitude might have become sufficient to perturb the reversed granulation pattern, causing a lower anticorrelation with the continuum and a periodic behaviour (more details in Janssen \& Cauzzi 2006).

The definition of the geometrical height for the phenomena is a challenge, since the surface of optical depth unity at any given wavelength is highly corrugated. Classical analysis employing semi-empirical 1D models give a formation height of about $200 \mathrm{~km}$ for the FeI core, where modern 3D hydrodynamical simulations display the reversal of granular and intergranular temperature (Nordlund 1985; Wedemeyer et al. 2004).

\section{References}

Cavallini, F. 2006, Solar Phys. 236(2), 415

Janssen, K. \& Cauzzi, G. 2006, A\& A 450, 365

Mihalas, B.W. \& Toomre, J. 1981, ApJ 249, 349

Nordlund, A. 1985, Solar Phys. 100, 209

Rutten, R.J., de Wijn, A.G. \& Sütterlin, P. 2004, A\&A 416, 333

Uitenbroek, H. 1989, A\& $A$ 213, 360

Wedemeyer, S., Freytag, B., Steffen, M., Ludwig, H.-G. \& Holweger, H. 2004, A\&A 414, 1121 